\title{
Study of physico-chemical properties, detection and toxicity study of organic compounds from effluent of MIDC Thane and GIDC Ankleshwar industrial zone
}

\author{
Prashant Bhimrao Koli ${ }^{1}$ D $\cdot$ Kailas Haribhau Kapadnis ${ }^{2} \cdot$ Uday Gangadhar Deshpande $^{1}$
}

Received: 14 May 2018 / Accepted: 2 October 2018 / Published online: 10 October 2018

(c) The Author(s) 2018

\begin{abstract}
The anthropological activities and huge industrialization to fulfil needs of mankind are making the remarkable and disastrous effect on aquatic life and responsible for severe pollution. The research deals with the identification and detection of organic pollutants present in industrial effluent by FTIR and GC-MS techniques. The samples were collected from paint, textile and dyes industries of MIDC Thane and GIDC Ankleshwar situated in western zone of India and recognized to be the most polluted cities in Asia. The samples were collected by standard operating procedure and then operated for extraction in ether so as to dissolve maximum organic compounds. These samples after extraction sealed in airtight glass vessel and used for FTIR and GC-MS analysis. The large number of organic compounds was detected by GC-MS analysis, whereas the presence of different functional groups of organic pollutants confirmed by FTIR analysis. The physico-chemical analysis was performed for effluent samples to know the different chemical factors associated with aqua samples. The statistical analysis of collected data was carried out; it comprises the mean, standard deviation, standard errors, Pearson correlation constants and regression analysis. The calculated results compared with WHO standards and water quality index were calculated. Large number of organic and aromatic compounds identified from GC-MS data and their toxicity is discussed.
\end{abstract}

Keywords Chemical toxicity $\cdot$ Effluent $\cdot$ Extraction $\cdot$ FTIR $\cdot$ GCMS $\cdot$ Physico-chemical $\cdot$ WQI

Prashant Bhimrao Koli

prashantkoli005@gmail.com

Kailas Haribhau Kapadnis

khkapadnis@yahoo.co.in

Uday Gangadhar Deshpande

Drugdeshpande@gmail.com

1 Research Centre in Chemistry and PG Department of Chemistry, Pratap College of Arts, Science

and Commerce, Affiliated to North Maharashtra University, Amalner, Taluka -Amalner, Jalgaon, Maharshtra 425401, India

2 Research Centre in Chemistry and PG Department of Chemistry, Loknete Vyankatrao Hiray Arts, Science and Commerce, College, Affiliated to SPPU, Pune (MH), Panchavati, Nashik 422003, India

\section{Introduction}

The rapid urbanization and immense industrialization is making a mark influence on global environment, especially water, air and land pollution. The major pollution augmenting unit is a water pollution. In general, the water is considered to be polluted when its quality impaired by several anthropological activities (Birjandi et al. 2016). Some of the major sources responsible for water pollution are excess discharge of untreated sewage from various industries, waste water production due to anthropological activities released in the rivers, costal area (Sharma et al. 2017; Kumar and Krishna 2017). The major contribution for water pollution reported due to untreated effluent of drugs, dyes, chemicals, pharmaceutical industries and nuclear waste, which are found to be huge pestilent. As most of the major pathogenic diseases, hazards and dermatological problems, are recognized due to consumption of polluted water, it is whole onus of industries that the effluent must be amend before subjecting to the discharge in various water sources (Wang et al. 2017; Nirgude et al. 2013).

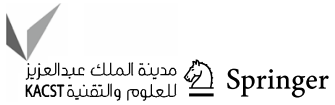


In undeveloped country like India, water pollution is a major issue because of polluted waste discharged in water streams may effect on economical budget if processed for sewage treatment. Hence, majority of industries released their sewage without further process and contributing to make the water contamination. However, most of the chemical industries take enough care while releasing the waste to the water streams, but this number is less and thus enhancing the pollution (Kumar et al. 2012). The pollutants present in water contain a large number of organic and inorganic contaminants which are unnecessary, producing pestilent and fatal effects on human health as well as aquatic life. These organic and inorganic pollutants as well as different pathogenic organism make the quality of water squalor. Most of the researchers are coalesce to defeat this problem of water pollution to serve the mankind better (Hladchenko et al. 2017).

The cities like Ankleshwar and Thane industrial found to be the most polluted according to the global pollution survey. Both the cities covered large industrial area and becoming a native place for most of the pharmaceutical, drugs, dyes, paint, textiles and API manufacturing industries (Mishra and Soni 2016; Mostafa 2015). These various drugs, dyes and paint industries synthesizes huge quantity of organic products every year. Most of the products manufactured by industries are valuable to the society and domestic use, but at the same time waste by-products and waste water which is left after synthesizing these products is a major environmental threat. This waste water indiscriminately flowed in the water streams lead to an inception of water pollution imperceptibly (Ouasif et al. 2013). Most of the researchers worked on this type of serious issue and published their view and facts related to waste water from these industrial zones (Patil and Shrivastava 2016).

The effluent samples were collected from paint, Textile and dye industries. The effluent discharged by them contains astonishing contaminants which ruffles quality of water. It is previously anticipated that these industries utilize large number of chemicals that are organic in nature. Even though the finished products synthesized by these industries are probably organic in nature. The inception of water pollution is started from these organic compounds; nevertheless, these industries discharged their waste after synthesis of their products, dying, processing, etc., in the river stream or costal region (Ong et al. 2011). Textile and paint industries also utilize different colours, dyes and pigments which are organic in nature (CPCB 2016; Chandran 2016).

In the present research, an attempt has made to identify and detect the different organics that are probably present in effluent sources. Also, the physico-chemical analysis of collected water samples was examined. Finally, the results obtained from physico-chemical analysis subjected for water quality index comparison. The toxicity of organic compound detected by GC-MS has been discussed.

\section{Materials and methods}

Diethyl ether $\left[\left(\mathrm{C}_{2} \mathrm{H}_{5}\right)_{2} \mathrm{O}\right]$ AR grade purchased from Merck, India. All the effluent samples (7 samples) were collected from MIDC Thane, India and GIDC, Ankleshwar, India. Solvent extraction technique was used to separate the organic contaminants present in the collected effluent samples.

\section{Sampling of effluents}

Three samples were collected from dyes and textile industries, from waste water effluent outlet Ankleshwar GIDC (Gujarat Industrial Development Co-operation, Ankleshwar), and four samples were collected from various paint and dye industries of Thane MIDC (Maharashtra Industrial Development Co-operation, Thane). Collected samples were stored in glass bottles previously washed with acetone to remove all the organics and further with nitric acid as well as by using double-distilled water, to remove all the metallic and other inorganic content previously present. All the containers then dried and kept in hot air oven for three hours at $120^{\circ} \mathrm{C}$. All the extracted samples were preserved in refrigerator for further analysis (Patil and Shrivastava 2013; Mahajan and Shrivastava 2013). The labelling of the collected samples is shown in Table 1.

\section{Sample preparation for FTIR analysis}

All the samples were extracted in diethyl ether; the aqueous and organic layer was separated by use of solvent extraction technique in separating funnel. After extraction, organic layer was stored in airtight glass vessel and used for FTIR characterization (Ladwani et al. 2016).

\section{Sample preparation for GC-MS analysis}

Similar extraction procedure was followed for GC-MS analysis, i.e. solvent extraction technique (Helaleh et al. 2001). After extraction, the samples AG2, TM5 and TM6 were used for GC-MS analysis (Thermo Scientific TSQ 8000).

Table 1 Sampling locations and source of sampling

\begin{tabular}{llll}
\hline S. no. & Sample name & Sampling point & Sampling source \\
\hline 01 & AG1 & Ankleshwar GIDC & Dyes and textiles \\
02 & AG2 & Ankleshwar GIDC & Dyes and textiles \\
03 & AG3 & Ankleshwar GIDC & Dyes and textiles \\
04 & TM4 & Thane MIDC & Paint industry \\
05 & TM5 & Thane MIDC & Paint industry \\
06 & TM6 & Thane MIDC & Dyes \\
07 & TM7 & Thane MIDC & Dyes \\
\hline
\end{tabular}




\section{Detection of organics by FTIR}

The main purpose of FTIR study is to investigate the possible organics present in the collected effluents. All the samples were extracted in diethyl ether. The labelled samples (Table 1) were sent for FTIR studies to detect the possible functional groups that are present in collected effluent samples (Table 2).

\section{Detection and identification of organics by GC-MS}

The samples AG-2, TM-5 and TM-6 were extracted in diethyl ether. These extracted samples were analysed by
GC-MS (Thermo Scientific TSQ 8000). The GC-MS data from which speculated organics obtain their corresponding spectrum are depicted in Table 3. The imperceptibly concentration is detected by GC-MS. The toxic, fatal and environmental hazards of selected compounds are discussed in results and discussion section (Srebrenkoska et al. 2014; Kotowska et al. 2012).

\section{Statistical analysis of data obtained from physico-chemical results}

Table 4.

Table 2 Detected organic functional groups in collected wastewater sample

\begin{tabular}{|c|c|c|c|c|c|}
\hline S. no. & Frequency in $\mathrm{cm}^{-1}$ & Interpretation (functional group) & S. no. & Frequency in $\mathrm{cm}^{-1}$ & Interpretation (functional group) \\
\hline Sample AG1 & & & Sample TM4 & & \\
\hline 01 & 2974.23 & Alkane $\mathrm{C}-\mathrm{H}$ & 01 & $2924(2850-2975)$ & Alkane $\mathrm{C}-\mathrm{H}$ \\
\hline 02 & 2862.36 & $\mathrm{C}-\mathrm{H}$ stretching freq in $-\mathrm{CH}_{3}$ & 02 & 2854.65 & $\mathrm{C}-\mathrm{H}$ stretching freq in $-\mathrm{CH}_{3}$ \\
\hline 03 & 1975.11 & $\mathrm{C}=\mathrm{O}$ stretch in acid/aldehyde & 03 & 1712.79 & $\mathrm{C}=\mathrm{O}$ stretch in acid/aldehyde \\
\hline 04 & 1450.47 & $\mathrm{C}-\mathrm{H}$ bending in $-\mathrm{CH}_{2}$ & 04 & 1458.18 & $\mathrm{C}-\mathrm{H}$ bending in $-\mathrm{CH}_{2}$ \\
\hline 05 & 1381.03 & $\mathrm{C}-\mathrm{O}$ stretching in benzoate & 05 & 1265.30 & \\
\hline 06 & 1288.45 & $\mathrm{C}-\mathrm{O}-\mathrm{C}$ stretch in ether (broad) & 06 & 740.67 & Monosubstituted aromatics \\
\hline 07 & 1122.57 & $\mathrm{C}-\mathrm{N}$ stretching in sec. amines & - & - & - \\
\hline 08 & 929.69 & Ethylene & - & - & - \\
\hline 09 & 840.96 & Tetra-substituted benzene & - & - & - \\
\hline 10 & 752.24 & Monosubstituted aromatic ring & - & - & - \\
\hline Sample AG2 & & & Sample TM5 & & \\
\hline 01 & 2970.38 & Alkane $\mathrm{C}-\mathrm{H}$ & 01 & 2924.09 & Alkane $\mathrm{C}-\mathrm{H}$ \\
\hline 02 & 2924.09 & & 02 & 2862.63 & $\mathrm{C}-\mathrm{H}$ stretching freq in $-\mathrm{CH}_{3}$ \\
\hline 03 & 2862.36 & $\mathrm{C}-\mathrm{H}$ stretching freq in $-\mathrm{CH}_{3}$ & 03 & 1728.22 & $\mathrm{C}=\mathrm{O}$ stretch in acid/aldehyde \\
\hline 04 & 1728.22 & $\mathrm{C}=\mathrm{O}$ stretch in acid/aldehyde & 04 & 1597.06 & $\mathrm{C}=\mathrm{C}$ frequency for alkene \\
\hline 05 & 1450.47 & $\mathrm{C}-\mathrm{H}$ bending in $-\mathrm{CH}_{2}$ & 05 & 1458.18 & $\mathrm{C}-\mathrm{H}$ bending in $\mathrm{CH} 2$ \\
\hline 06 & 1373.32 & & 06 & 1381.03 & \\
\hline 07 & 1126.43 & $\mathrm{C}-\mathrm{N}$ stretching in sec. amines & 07 & 1273.02 & $\mathrm{C}-\mathrm{O}-\mathrm{C}$ stretch in ether (broad) \\
\hline 08 & 840 & Tetra-substituted benzene & 08 & 1126.43 & $\mathrm{C}-\mathrm{N}$ stretching in sec.amines \\
\hline & - & - & 09 & 1064.71 & $\mathrm{C}-\mathrm{O}$ stretching freq in alcohols/ethers \\
\hline & - & - & 10 & 740.67 & Monosubstituted aromatics \\
\hline Sample AG3 & & & Sample TM6 & & \\
\hline 01 & 3425.58 & O-H stretch (broad) & 01 & 2970.38 & Alkane $\mathrm{C}-\mathrm{H}$ \\
\hline 02 & 3001.24 & Chelate $\mathrm{H}$ bridge $(\mathrm{O}-\mathrm{H})$ & 02 & 2862.36 & $\mathrm{C}-\mathrm{H}$ stretching freq in $-\mathrm{CH}_{3}$ \\
\hline 03 & 2877.81 & $\mathrm{C}-\mathrm{H}$ bending in $-\mathrm{CHO}$ & 03 & 1450.47 & $\mathrm{C}-\mathrm{H}$ bending in $-\mathrm{CH}_{2}$ \\
\hline 04 & 1458.18 & $\mathrm{O}=\mathrm{S}=\mathrm{O}$ stretch & 04 & 1373.32 & \\
\hline 05 & 1350.17 & & 05 & 1126.43 & $\mathrm{C}-\mathrm{N}$ stretching in sec.amines \\
\hline 06 & 1296.16 & $\mathrm{C}-\mathrm{O}-\mathrm{C}$ stretch in ether (broad) & 06 & 925.83 & $\equiv \mathrm{CH}$ bending frequency \\
\hline 07 & 1249.87 & $\mathrm{C}-\mathrm{H}$ out of plane bending & 07 & 840.96 & Tetra-substituted benzene \\
\hline 08 & 1111.10 & Anhydride $\mathrm{C}=\mathrm{O}$ stretch & - & - & - \\
\hline 09 & 941.26 & $\equiv \mathrm{CH}$ bending frequency & - & - & - \\
\hline 10 & 756.10 & Monosubstituted aromatics & - & - & - \\
\hline
\end{tabular}


Table 3 Probable organic compounds detected from collected effluent samples AG2, TM5 and TM6 by GC-MS technique

\begin{tabular}{|c|c|c|c|c|c|}
\hline S. no. & Name of compound & RT & Molecular formula & $\begin{array}{l}\text { Molecular } \\
\text { weight in } \mathrm{g}\end{array}$ & CAS number \\
\hline \multicolumn{6}{|c|}{ Sample AG2 } \\
\hline 01 & 4-Methyl-3-hexanol & 4.83 & $\mathrm{C}_{7} \mathrm{H}_{16} \mathrm{O}$ & 116 & $615-29-2$ \\
\hline 02 & 2,3-Dimethyl-2-butanol & 4.83 & $\mathrm{C}_{6} \mathrm{H}_{14} \mathrm{O}$ & 102 & $594-60-5$ \\
\hline 03 & 3-Heptanol & 4.83 & $\mathrm{C}_{7} \mathrm{H}_{16} \mathrm{O}$ & 116 & $589-82-2$ \\
\hline 04 & Hexadecane & 11.49 & $\mathrm{C}_{16} \mathrm{H}_{34}$ & 226 & $544-76-3$ \\
\hline 05 & 2,6,10-Trimethyl tetradecane & 11.49 & $\mathrm{C}_{17} \mathrm{H}_{36}$ & 240 & $14905-56-7$ \\
\hline 06 & Heptadecane & 11.49 & $\mathrm{C}_{17} \mathrm{H}_{36}$ & 240 & $629-78-7$ \\
\hline 07 & 2,6-Dihexadecanoate-1-(+)-ascorbic acid & 14.13 & $\mathrm{C}_{38} \mathrm{H}_{68} \mathrm{O}_{8}$ & 652 & $28474-90-0$ \\
\hline 08 & $n$-Hexadecanoic acid & 14.33 & $\mathrm{C}_{16} \mathrm{H}_{32} \mathrm{O}_{2}$ & 256 & $57-10-3$ \\
\hline 09 & Palmitic acid anhydride & 14.33 & $\mathrm{C}_{32} \mathrm{H}_{62} \mathrm{O}_{3}$ & 494 & $623-65-4$ \\
\hline 10 & 3-Ethyl-5-(2-ethylbutyl)-octadecane & 14.38 & $\mathrm{C}_{26} \mathrm{H}_{54}$ & 366 & $55282-12-7$ \\
\hline 11 & 3,5-Dehydro-6-methoxy-pivalate-cholest-22-ene-21-ol & 14.38 & $\mathrm{C}_{33} \mathrm{H}_{54} \mathrm{O}_{3}$ & 498 & NA \\
\hline 12 & 2,6,10-Trimethyl tetradecane & 14.38 & $\mathrm{C}_{17} \mathrm{H}_{36}$ & 240 & $14905-56-7$ \\
\hline 13 & 1-Monolinoleoylglycerol trimethylsilyl ether & 19.50 & $\mathrm{C}_{27} \mathrm{H}_{54} \mathrm{O}_{4} \mathrm{Si}_{2}$ & 470 & $54284-45-6$ \\
\hline 14 & 2,3-Bis(trimethylsilyl) oxylporpyl ester, $(z, z, z)-9,12,15$-octadecatrienoic acid & 19.50 & $\mathrm{C}_{27} \mathrm{H}_{52} \mathrm{O}_{4} \mathrm{Si}_{2}$ & 440 & $55521-22-7$ \\
\hline 15 & $\begin{array}{l}\text { 25-(Trimethylsilyl) oxy-(3a, 5Z, 7E)-9, 10-secocholesta-5, 7, } 10 \text { (19)-tiene-1-3- } \\
\text { diol }\end{array}$ & 19.50 & $\mathrm{C}_{30} \mathrm{H}_{52} \mathrm{O}_{3} \mathrm{Si}$ & 488 & $55759-94-9$ \\
\hline 16 & Ethyl iso-allocholate & 19.69 & $\mathrm{C}_{26} \mathrm{H}_{44} \mathrm{O}_{5}$ & 536 & NA \\
\hline 17 & (3a,5Z,7E)-9, 10-Secocholesta-5, 7, 10 (19)-tiene-3, 24,25-triol & 19.69 & $\mathrm{C}_{27} \mathrm{H}_{44} \mathrm{O}_{3}$ & 416 & 40013-87-4 \\
\hline 18 & 3-Acetoxy-7,8-epoxylanostan-11-ol & 19.69 & $\mathrm{C}_{32} \mathrm{H}_{54} \mathrm{O}_{4}$ & 502 & NA \\
\hline 19 & 4-en-3-one-stigmast & 21.11 & $\mathrm{C}_{29} \mathrm{H}_{48} \mathrm{O}$ & 412 & $1058-61-3$ \\
\hline 20 & Cypionate testosterone & 21.11 & $\mathrm{C}_{27} \mathrm{H}_{40} \mathrm{O}_{3}$ & 412 & $58-20-8$ \\
\hline 21 & 4-en-3-one-cholest & 21.11 & $\mathrm{C}_{27} \mathrm{H}_{44} \mathrm{O}$ & 384 & $601-57-0$ \\
\hline \multicolumn{6}{|c|}{ Sample TM5 } \\
\hline 22 & Nonane & 4.69 & $\mathrm{C}_{9} \mathrm{H}_{20}$ & 128 & $111-84-2$ \\
\hline 23 & Decane & 4.69 & $\mathrm{C}_{10} \mathrm{H}_{22}$ & 142 & $124-18-5$ \\
\hline 24 & 2,4-Dimethyl hexane & 4.69 & $\mathrm{C}_{8} \mathrm{H}_{18}$ & 114 & $589-43-5$ \\
\hline 25 & 1-Ethyl-3-methyl benzene & 5.69 & $\mathrm{C}_{9} \mathrm{H}_{12}$ & 120 & $620-14-4$ \\
\hline 26 & 1-Ethyl-2-methyl benzene & 5.69 & $\mathrm{C}_{9} \mathrm{H}_{12}$ & 120 & $611-14-3$ \\
\hline 27 & 1,2,4-Trimethyl benzene & 5.69 & $\mathrm{C}_{9} \mathrm{H}_{12}$ & 120 & $95-63-6$ \\
\hline 28 & 1,2,3-Trimethyl benzene & 5.82 & $\mathrm{C}_{9} \mathrm{H}_{12}$ & 120 & $526-73-8$ \\
\hline 29 & 3,5-Dimethyl octane & 6.29 & $\mathrm{C}_{10} \mathrm{H}_{22}$ & 142 & $15869-93-9$ \\
\hline 30 & 4,6-Dimethyl undecane & 6.29 & $\mathrm{C}_{13} \mathrm{H}_{28}$ & 184 & $17312-82-2$ \\
\hline 31 & 4-Methyl-decane & 6.64 & $\mathrm{C}_{11} \mathrm{H}_{24}$ & 156 & $2847-72-5$ \\
\hline 32 & 2-Ethylhexyl ester, trichloroacetic acid & 6.64 & $\mathrm{C}_{10} \mathrm{H}_{17} \mathrm{Cl}_{3} \mathrm{O}_{2}$ & 275 & $16397-79-8$ \\
\hline 33 & 5-Ethyl-2-methyl heptane & 6.64 & $\mathrm{C}_{10} \mathrm{H}_{22}$ & 142 & $13475-78-0$ \\
\hline 34 & Undecane & 7.89 & $\mathrm{C}_{11} \mathrm{H}_{24}$ & 156 & $1120-21-4$ \\
\hline 35 & Dodecane & 7.89 & $\mathrm{C}_{12} \mathrm{H}_{26}$ & 170 & $112-40-3$ \\
\hline 36 & 4,5-Dimethyl nonane & 7.89 & $\mathrm{C}_{11} \mathrm{H}_{24}$ & 156 & $17302-23-7$ \\
\hline 37 & (Z)-9,17-Octadecadienal & 20.34 & $\mathrm{C}_{18} \mathrm{H}_{32} \mathrm{O}$ & 264 & $56554-35-9$ \\
\hline 38 & 7,11-Hexadecadienal & 20.34 & $\mathrm{C}_{16} \mathrm{H}_{28} \mathrm{O}$ & 236 & NA \\
\hline 39 & 12-Methyl-E,E-2,13-octadecadienal-1-ol & 20.34 & $\mathrm{C}_{19} \mathrm{H}_{36} \mathrm{O}$ & 280 & NA \\
\hline \multicolumn{6}{|c|}{ Sample TM6 } \\
\hline 40 & 5-Methyl-3-hexanol & 4.84 & $\mathrm{C}_{7} \mathrm{H}_{15} \mathrm{O}$ & 115 & $615-29-2$ \\
\hline 41 & Phenol & 5.43 & $\mathrm{C}_{6} \mathrm{H}_{6} \mathrm{O}$ & 94 & $108-95-2$ \\
\hline 42 & $p$-Hydroxy phenyl phosphonic acid & 5.43 & $\mathrm{C}_{6} \mathrm{H}_{7} \mathrm{O}_{4} \mathrm{P}$ & 174 & $33795-18-5$ \\
\hline 43 & Carbamic acid, phenyl ester & 5.43 & $\mathrm{C}_{7} \mathrm{H}_{7} \mathrm{NO}_{2}$ & 137 & $622-46-8$ \\
\hline 44 & 2,6-Dimethyl quinoline & 10.17 & $\mathrm{C}_{11} \mathrm{H}_{11} \mathrm{~N}$ & 157 & $877-43-0$ \\
\hline 45 & 2,8-Dimethyl quinoline & 10.17 & $\mathrm{C}_{11} \mathrm{H}_{11} \mathrm{~N}$ & 157 & $1463-17-8$ \\
\hline
\end{tabular}


Table 3 (continued)

\begin{tabular}{|c|c|c|c|c|c|}
\hline S. no. & Name of compound & RT & Molecular formula & $\begin{array}{l}\text { Molecular } \\
\text { weight in } g\end{array}$ & CAS number \\
\hline 46 & 4,8-Dimethyl quinoline & 10.17 & $\mathrm{C}_{11} \mathrm{H}_{11} \mathrm{~N}$ & 157 & $13362-80-6$ \\
\hline 47 & 7-Methoxy-2,2,4,8-tetramethyl tricyclo[5.3.1.0(4,11)] undecane & 10.37 & $\mathrm{C}_{16} \mathrm{H}_{28} \mathrm{O}$ & 236 & NA \\
\hline 48 & 2-Acetyl-3,3-dimethyl-2-(3-oxo-1-butenyl)-(E)-cyclopentanone & 10.37 & $\mathrm{C}_{13} \mathrm{H}_{18} \mathrm{O}_{3}$ & 222 & $70412-49-6$ \\
\hline 49 & 3-(1,1-Dimethylethyl)-4-methoxy, phenol & 10.37 & $\mathrm{C}_{11} \mathrm{H}_{16} \mathrm{O}_{2}$ & 180 & $88-32-4$ \\
\hline 50 & 2,6-bis(1,1-dimethylethyl)-2,5-cyclohexadiene-1,4-dione & 10.44 & $\mathrm{C}_{14} \mathrm{H}_{20} \mathrm{O}_{2}$ & 220 & $719-22-2$ \\
\hline 51 & 2,5-di-tert-butyl-1,4-benzoquinone & 10.44 & $\mathrm{C}_{14} \mathrm{H}_{20} \mathrm{O}_{2}$ & 220 & $2460-77-7$ \\
\hline 52 & Furan-5-yl-1(5,6,7,8-tetrahydro-2,8,8-trimethyl-4H-cyclohepta, ethanone & 10.44 & $\mathrm{C}_{14} \mathrm{H}_{20} \mathrm{O}_{2}$ & 220 & $71596-88-8$ \\
\hline 53 & Butylated hydroxytoluene & 10.76 & $\mathrm{C}_{15} \mathrm{H}_{24} \mathrm{O}$ & 220 & $128-37-0$ \\
\hline 54 & 4,6-di(1,1-dimethylethyl)-2-methyl-phenol & 10.76 & $\mathrm{C}_{15} \mathrm{H}_{24} \mathrm{O}$ & & $616-55-7$ \\
\hline 55 & 2,4,6-tris(1-methylethyl)-Phenol & 10.76 & $\mathrm{C}_{15} \mathrm{H}_{24} \mathrm{O}$ & 220 & 2934-07-8 \\
\hline 56 & Heptadecane & 11.48 & $\mathrm{C}_{17} \mathrm{H}_{36}$ & 240 & $629-78-7$ \\
\hline
\end{tabular}

$N A$ not applicable; $C A S$ chemical abstracts service

Table 4 Physico-chemical properties of collected industrial effluent samples

\begin{tabular}{|c|c|c|c|c|c|c|c|}
\hline $\begin{array}{l}\text { Parameter } \\
\text { Unit }\end{array}$ & $\begin{array}{l}\mathrm{PH} \\
(\mu \mathrm{mho} / \mathrm{cm})\end{array}$ & $\begin{array}{l}\text { Electrical conductivity } \\
(\mu \mathrm{mho} / \mathrm{cm})\end{array}$ & $\begin{array}{l}\text { Sulphate } \\
(\mathrm{mg} / \mathrm{L})\end{array}$ & $\begin{array}{l}\text { Chloride } \\
(\mathrm{mg} / \mathrm{L})\end{array}$ & $\begin{array}{l}\mathrm{COD} \\
\left(\mathrm{mg} / \mathrm{L} \mathrm{O}_{2}\right)\end{array}$ & $\begin{array}{l}\mathrm{BOD} \\
\left(\mathrm{mg} / \mathrm{L} \mathrm{O}_{2}\right)\end{array}$ & $\begin{array}{l}\text { TDS } \\
(\mathrm{mg} / \mathrm{L})\end{array}$ \\
\hline AG1 & 8.2 & 398 & 91.60 & 262 & 1610 & 536 & 1590 \\
\hline AG2 & 7.8 & 402 & 82 & 300 & 1460 & 486 & 1620 \\
\hline AG3 & 7.7 & 500 & 76 & 387 & 1570 & 523 & 1480 \\
\hline TM4 & 8.5 & 398 & 93.50 & 281.50 & 1760 & 586 & 1805 \\
\hline TM5 & 7.5 & 402 & 86.42 & 310 & 1350 & 450 & 1745 \\
\hline TM6 & 7.4 & 500 & 72.83 & 418 & 1465 & 488 & 1654 \\
\hline TM7 & 7.2 & 530 & 80.00 & 497 & 1580 & 526 & 1970 \\
\hline Mean & 7.26 & 447.14 & 83.19 & 350.78 & 1542.14 & 513.57 & 1694.85 \\
\hline S.D & 0.95 & 40.1246 & 7.7233 & 85.76 & 131.58 & 43.68 & 160.61 \\
\hline S.E & 0.36 & 15.16 & 2.92 & 32.42 & 49.73 & 16.51 & 60.70 \\
\hline WHO & $6.5-9.5$ & 1400 & 500 & 250 & - & - & 1000 \\
\hline US-EPA & $6.5-8.5$ & 250 & 250 & 250 & - & - & 500 \\
\hline
\end{tabular}

$S D$ standard deviation; $S E$ standard error

\begin{tabular}{|c|c|c|c|c|c|c|c|}
\hline \multirow[t]{2}{*}{ Parameters } & \multicolumn{7}{|c|}{ Correlation coefficient $(r)$ of different parameters } \\
\hline & $\mathrm{pH}$ & $\mathrm{EC}$ & Sulphates & Chlorides & COD & BOD & TDS \\
\hline $\mathrm{PH}$ & 1 & & & & & & \\
\hline Electrical conductivity & -0.9514 & 1 & & & & & \\
\hline Sulphates & 0.81 & -0.797 & 1 & & & & \\
\hline Chlorides & -0.9758 & 0.9603 & -0.7435 & 1 & & & \\
\hline COD & 0.2916 & -0.239 & 0.4545 & -0.1145 & 1 & & \\
\hline BOD & 0.2917 & -0.0237 & 0.4546 & -0.1146 & 1 & 1 & \\
\hline TDS & -0.2684 & 0.1763 & 0.2143 & 0.4009 & 0.1654 & 0.1649 & 1 \\
\hline
\end{tabular}

Table 5 Pearson correlation coefficient $(r)$ of different physico-chemical parameters for waste water effluent samples 
Table 6 Water quality index data for collected industrial effluents

\begin{tabular}{|c|c|c|c|c|c|c|c|c|c|c|}
\hline \multirow[t]{2}{*}{ Parameter } & \multicolumn{10}{|c|}{ Tested value (Vn) } \\
\hline & AG1 & AG2 & AG3 & TM4 & TM5 & TM6 & TM7 & $(\mathrm{Wn})$ & $\mathrm{Sn}$ & V10 \\
\hline $\mathrm{pH}$ & 8.2 & 7.8 & 7.7 & 8.5 & 7.5 & 7.4 & 7.2 & 0.219 & 7.5 & 7.0 \\
\hline $\mathrm{EC}$ & 398 & 402 & 500 & 398 & 402 & 500 & 530 & 0.371 & 300 & 0 \\
\hline Sulphates & 91.60 & 82 & 76 & 93.50 & 86.42 & 72.83 & 80 & 0.01236 & 500 & 0 \\
\hline Chlorides & 262 & 300 & 387 & 281.5 & 310 & 418 & 497 & 0.0074 & 250 & 0 \\
\hline TDS & 1590 & 1620 & 1480 & 1805 & 1745 & 1654 & 1970 & 0.0037 & 1000 & 0 \\
\hline$\sum \mathrm{Qn}$ & 695.86 & 740 & 624 & 743.80 & 549.79 & 593.82 & 628.46 & - & - & - \\
\hline$\sum \mathrm{Wn} \cdot \mathrm{Qn}$ & 103.65 & 88.26 & 93.52 & 116.40 & 73.38 & 81.37 & 76.69 & - & - & - \\
\hline WQI & 168 & 143 & 152.45 & 189.75 & 119.62 & 132.65 & 125.26 & - & - & - \\
\hline
\end{tabular}

Sn, std. value; V10, ideal value; Qn, quality rating; Wn, unit weight; WQI, water quality index

\section{Pearson correlation coefficient $(r)$ data of different parameters}

Table 5.

\section{Water quality index}

Table 6.

\section{Results and discussions}

\section{Physico-chemical properties}

(a) $p H$ The negative proton ions are universal species that decide the acidic and basic condition of aqua solution. The species strongly affect different properties, characteristics of water. The major reliable contribution made by the authentic institutes such as WHO, USEPA to control important water parameters (Elango et al. 2017). The present investigation was compared against the standard data of these institutes. Here, the maximum value of protonic ions was shown by sample AG1, i.e. 8.2 (slightly alkaline, MIDC Thane), while the least value was shown by sample TM7, i.e. 5.76 (Acidic, GIDC Ankleshwar). All the samples comprise the given range prescribed by the standard data.

(b) Electrical conductivity Total dissolved salts are generally responsible for high values of electrical conductivity (Manikandan et al. 2015). Values of electrical conductivity were in the range of $398-530 \mu \mathrm{m} / \mathrm{cm}$. The highest value was observed for sample TM7, probably due to the presence of dissolved inorganic salts in the collected sample. The electrical conductivity data pass the permissible limit prescribed by the WHO and USEPA standards.

(c) Sulphates Probably sulphates are present in water streams due to utilization of sulphonates and sulphu- ric acid for various industrial processes such as tanning. Most of the time magnesium and calcium sulphates are responsible for permanent water hardness. The sulphates data obtained for all the effluent sample are listed in table 4 . This data matches within the priscribed limit of WHO and US-EPA (Periyasamy and Rajan 2009).

(d) Chlorides Chloride ions originate probably from sewage, industrial effluent, several natural sources, in certain cases due to urban activities such as saline intrusion, de-icing salt process, etc. In all the collected samples, the chloride values were found in the range of $262-497 \mathrm{mg} / \mathrm{L}$. The values of chloride ion found to be very high and beyond the decided level of WHO.

(e) $B O D$ The biological oxygen demand is found to be higher in all the samples indicating the more percentage of bacterial content utilizing the oxygen, probably due to the presence of higher organics content in the sample.

(f) $C O D$ The higher values of $C O D$ are attributed to more carbon containing waste present in all the samples, because all the samples which are collected are from organic source (Brahmbhatt and Pandya 2015).

(g) TDS The total dissolved solids are the organic contaminants and inorganic insoluble, suspended particles, sulphates, phosphates, sodium chloride, etc., are present due to which the water quality is ruffled. The TDS value limited by authorized institutes is said to be $1000 \mathrm{mg} / \mathrm{L}$, while all the samples have astonishing TDS values (Rawway et al. 2016).

\section{Correlation coefficient}

Correlation coefficient was calculated to know the relationship between two variables. The positive relationship is assumed when the value expected is 1 ; the strong negative relationship is expected when $R$ has a value -1 . The net zero value indicates no relationship between two 
Table 7 Water quality index report status

\begin{tabular}{lcl}
\hline S. no. & $\begin{array}{l}\text { Water quality } \\
\text { index range }\end{array}$ & Quality of water \\
\hline $\begin{array}{l}\text { Water quality index legend } \\
01\end{array}$ & $90-100$ & Unsuitable for potable purpose \\
02 & $70-89$ & Very poor quality water \\
03 & $50-70$ & Ruffled water quality \\
04 & $25-50$ & Good quality water \\
05 & $0-25$ & Excellent quality water \\
\hline
\end{tabular}

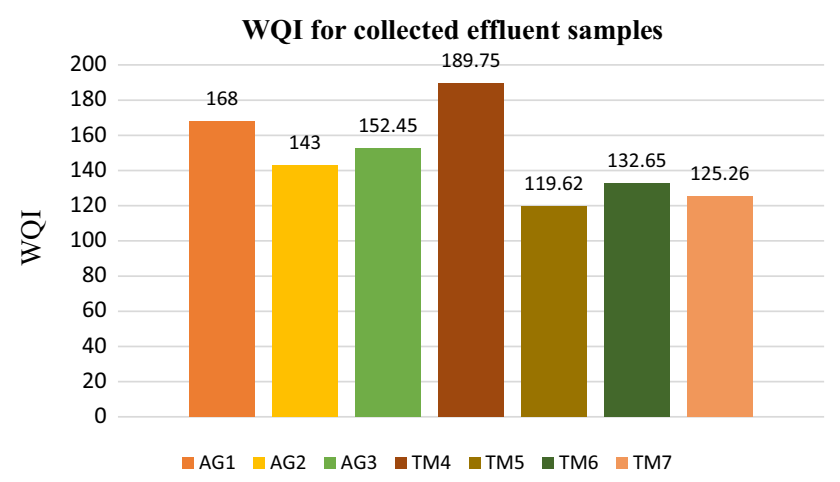

Fig. 1 Diagrammatic representation of calculated water quality index (WQI) for all the collected effluent samples

variables. The correlation coefficient was calculated by using formula

$\mathbf{r}=\frac{\mathbf{n}\left(\sum x y\right)-\left(\sum x\right)\left(\sum y\right)}{\left[n \sum X^{2}-\left(\sum x\right)^{2}\right]\left[n \sum Y^{2}-\left(\sum y\right)^{2}\right]}$

The correlation coefficient values for selected variables depicted in Table 5 show good agreement of values for present variables (Saxena and Saxena 2015; Nagaraju et al. 2017). The correlation coefficient values are depicted in Table 5.

\section{Water quality index}

Water quality index is 100-point scale of water parameters considered for physico-chemical analysis. In the present discussion, five parameters are examined for water quality index. The water quality index was found to be very high for all the collected effluent samples; all the effluent samples are unsuitable for potable and other purposes due to high WQI. The standard water quality index report is shown in Table 7. The diagrammatic representation of calculated water quality index of all the collected effluent samples is summarized in Fig. 1.
Toxicity of selected compounds detected by GC-MS in effluents

As the samples contain dissolved organics, these samples were analysed by GC-MS. The detected organics and their data obtained are as shown in Table 3. In this section, the toxicity of some selected organic compounds detected in GC-MS is discussed.

(a) 2-ethyl, hexyl ester trichloroacetic acid The compound in association with water (drinking or effluent) produces complex by-products disinfectants which are found to raise the risk of colon, rectal and bladder cancer in human (Kumar and Pandit 2012) and adverse effects on reproduction.

(b) 3,5-dehydro-6-methoxy-pivalate-cholest-22-ene-21-ol The compound was investigated for microbiological studies and showed toxic effects against the S. pyogenes organism that was found to be effective. This compound serves as an effecive reagent to inhibit the growth of most of pathogenic and non-pathogenic microorganisms (Singariya et al. 2016).

(c) 4,6-di(1,1-dimethylethyl)-2-methyl-phenol This is one of the toxic compounds detected in cigarette mainstream smoke (CMS), and if this compound is found in water streams then its free radicals are responsible for cardiovascular disease, (Smith et al. 2002) occupational and environmental lung diseases. Substituted phenols with electron releasing molecules can frame conceivably poisonous phenoxyl free radicals, and those substituted phenols with electron pulling back impact apply their lethality for the most part through lipophilicity (Vallyathan et al. 1998).

(d) Palmitic acid The major threat of suffering from cardiovascular diseases as well as enhancement of LDL level in the bloodstream may cause due to feeding of palmitic acid anhydride according to WHO reports. Oxidation of fats in the body and energy utilization rates may alter due to palmitic acid (Borsheim et al. 2006).

(e) Carbamic acid, phenyl ester The assessment of accessible poisonous quality information for fish, aquatic invertebrates and amphibian plants is moderate.

(f) 3-(1,1-dimethylethyl)-4-methoxy, phenol Most of the substituted phenol used as an antioxidant in food and fats, viz. BHA and BHA, is for preservation of food products (Davi and Gundi 1999). The toxicity of phenolic compounds can be explained by the substitution of this surface-active agent with new products, as polyethoxylated alcohols, with the substitution of the phenolic group, because of the problems correlated with toxicity accumulation and oestrogenic effects that this 
surfactant induced in some animals and organisms. Regular monitoring of phenolic compounds is essential due to their toxicity and bioaccumulation effects in animal and vegetable organisms (Kumar and Pacha 2015).

(g) Heptadecane However, carcinogenic reports, reproductive toxicity and genotoxicity chronic reports are not available for heptadecane, but several subchronic cases are reported for this compound.

(h) 3-acetoxy-7,8-epoxylanostan-11-ol The compound was investigated from plant extract of Hamelia patens using chloroform as extractor in the research work reported that 3-acetoxy-7,8-epoxylanostan-11-ol compound shows an antidepressant activity for animals (Surana and Wagh 2017).

(i) 4-en-3-one-stigmast This compound was extracted from bark of Anacardium occidentale (cashew), reported that the 4-en-3-one-stigmast shows hypoglycaemic effects (lowering of blood sugar level) in healthy dogs (Alexander-Lindo et al. 2004).

(j) 2,3-bis(trimethylsilyl)oxylporpyl ester, (z,z,z)-9,12,15octadecatrienoic acid The extraction of Diospyros Montana (Roxb.) reported the presence of this compound which shows some phytochemical effects (Bodele and Shahare 2018).

\section{Conclusions}

All the samples collected from Ankleshwar GIDC and Thane MIDC from paint and dyes industries were analysed by various physico-chemical parameters. The samples were found to be heavily contaminated, and some parameters not comply with standards reported by WHO and US-EPA. All the tested parameters such as BOD, COD, chlorides, sulphates and TDS were found to be very higher for AG1, AG2, AG3, TM4, TM5, TM6 and TM7 samples. Except $\mathrm{pH}$ and electrical conductivity passes the limit for all effluent samples. The water quality index report for all the samples was found to be extreme and not within the range of WQI; hence, it can be suggested that effluent must be treated by concern industries before discharging them into the river stream or nearby costal region so that contaminated water may become decontaminated. The infrared spectrum of the samples showed the corresponding functional groups of possible organic compounds in the samples as depicted in Table 2. The most important parameter analysed was GC-MS studied for the detection of organic contaminants. A wide range of organics were detected by this technique as the data as reported in Table 3. The most fatal and harmful compounds that can be harmful to the humans health as well as hazardous to the aquatic life are described such as, 2-ethyl, hexyl ester trichloroacetic acid,
3, 5-dehydro-6-methoxy-pivalate-cholest-22-ene-21-ol, 4, 6-di(1, 1-dimethylethyl)-2-methyl-phenol, palmitic acid, carbamic acid, phenyl ester, 3-(1, 1-dimethylethyl)-4-methoxy, phenol, heptadecane,3-acetoxy-7,8-epoxylanostan11-ol,4-en-3-one-stigmast,2,3-bis(trimethylsilyl)oxylporpyl ester, $(z, z, z)-9,12,15$-octadecatrienoic acid.

The large number of industries discharge harmful waste effluents into the water sterams that should be monitored by the concern industries. There must be some strong regulations for discharging such a toxic chemical in the form of effluent. The industries such as paint, dyes, chemical, pharmaceutical and API should take initiate to minimize the pollution by taking government help for waste management such as construction of adequate sanitary landfills sites, catalytic activities to convert harmful chemicals into less harmful chemicals or compounds, photocatalysis, recycling of waste water, conduction of epidemiological study in the polluted area and asses the health survey on the water consumers, etc.

In the present research, we tried to investigate waterrelated properties, but main objects were to envisage the organic compound present in the effluent sources. We discussed the toxicity of some organic compounds from the GC-MS data (listed in Table 3). However, it is highly impossible to seperate the individual organic compounds due to presence of large number compounds present in the water streams. Also, all the compounds detected by GC-MS are organic, and hence, their quantitative and qualitative separation is very complex task. Analysis of this type of effluent is very beneficial to the society and ecosystem. The detailed toxicity of all the listed compounds has been discussed in the results and discussion section.

Acknowledgement Authors are grateful to the Sophisticated Analytical Instrumentation facility (SAIF), Chandigarh, for providing GC-MS Facility and CIC, KTHM College, Nashik, for providing IR spectrums and Pratap College, Amalner, as well as L.V.H. College, Nashik, for providing necessary laboratory facilities.

Open Access This article is distributed under the terms of the Creative Commons Attribution 4.0 International License (http://creativeco mmons.org/licenses/by/4.0/), which permits unrestricted use, distribution, and reproduction in any medium, provided you give appropriate credit to the original author(s) and the source, provide a link to the Creative Commons license, and indicate if changes were made.

\section{References}

Alexander-Lindo RL, Morrison ES, Nair MG (2004) Hypoglycaemic effect of stigmast-4-en-3-one and its corresponding alcohol from the bark of Anacardium occidentale (cashew). Phytother Res 18(5):403-407

Birjandi N, Younesi H, Bahramifar N (2016) Treatment of wastewater effluents from paper-recycling plants by coagulation process and 
optimization of treatment conditions with response surface methodology. Appl Water Sci 6(4):339-348

Bodele SK, Shahare NH (2018) Phytochemical screening and GC-MS analysis of Diospyros Montana (Roxb.) root. Int J Res Pharmacol Pharmacother 7(2):100-107

B $ø$ rsheim E, Kien CL, Pearl WM (2006) Differential effects of dietary intake of palmitic acid and oleic acid on oxygen consumption during and after exercise. Metab Clin Exp 55(9):1215-1221

Brahmbhatt NH, Pandya KY (2015) Performance evaluation of effluent treatment plant and hazardous waste management of pharmaceutical industry of Ankleshwar. Adv Appl Sci Res 6(4):157-161

Chandran D (2016) A review of the textile industries waste water treatment methodologies. Int J Sci Eng Res 7:392-403

CPCB (2016) Report on environmental quality monitoring for assessment of comprehensive environmental pollution index (CEPI), for critically polluted area Ankleshwar in Gujarat

Davi ML, Gnudi F (1999) Phenolic compounds in surface water. Water Res 33(14):3213-3219

Elango G, Rathika G, Elango S (2017) Physico-chemical parameters of textile dyeing effluent and its impacts with case study. Int J Res Chem Environ 7(1):17-24

Helaleh MI, Takabayashi Y, Fujii S, Korenaga T (2001) Gas chromatographic-mass spectrometric method for separation and detection of endocrine disruptors from environmental water samples. Anal Chim Acta 428(2):227-234

Hladchenko LN, Matvyeyeva EL, Kipnis LS (2017) Assessment of wastewater toxicity after their treatment by biosorbents Ecolan-M and Econadin. J Water Chem Technol 39(5):294-298

Kotowska U, Biegańska K, Isidorov VA (2012) Screening of trace organic compounds in municipal wastewater by gas chromatography-mass spectrometry. Pol J Environ Stud 21(1):129-138

Kumar GV, Krishna KR (2017) Comparative study on the water quality status of Andra reservoir and Denkada anicut constructed on Champavati River, Vizianagaram, India. Appl Water Sci 7(3):1497-1504

Kumar PK, Pacha MM (2015) Assessment of phenolic compounds in the surface waters of Godavari Canal, Andhra Pradesh, India. Curr World Environ 10(1):338-342

Kumar JK, Pandit AB (2012) Drinking water disinfection techniques. CRC Press, Boca Raton

Kumar KR, Suman M, Archana S (2012) Water quality assessment of raw sewage and final treated water with special reference to waste water treatment plant Bhopal, MP, India. Res J Recent Sci $1: 185-190$

Ladwani KD, Ladwani KD, Ramteke DS, Deo S (2016) Detection and identification of organic compounds in wastewater of final effluent treatment plant by FTIR and GC-MS. J Adv Chem Sci 9:246-247

Mahajan SV, Shrivastava VS (2013) Identification of organic compounds in ground water samples by FTIR and GC-MS. Int J Chem Sci 11(3): 1582-1588

Manikandan P, Palanisamy PN, Baskar R, Sivakumar P, Sakthisharmila $P$ (2015) Physico chemical analysis of textile industrial effluents from Tirupur city, TN, India. Int J Adv Res Sci Eng 4(2):93-104

Mishra P, Soni R (2016) Analysis of dyeing and printing waste water of Balotara textile industries. Int J Chem Sci 14(4):1929-1938

Mostafa M (2015) Waste water treatment in textile Industries-the concept and current removal technologies. J Biodivers Environ Sci 7(1):501-525
Nagaraju A, Sreedhar Y, Thejaswi A, Sayadi MH (2017) Water quality analysis of the Rapur area, Andhra Pradesh, South India using multivariate techniques. Appl Water Sci 7(6):2767-2777

Nirgude NT, Shukla S, Venkatachalam A (2013) Physico-chemical analysis of some industrial effluents from Vapi industrial area, Gujarat, India. Rasayan J Chem 6:68-72

Ong ST, Keng PS, Lee WN, Ha ST, Hung YT (2011) Dye waste treatment. Water 3(1):157-176

Ouasif H, Yousfi S, Bouamrani ML, El Kouali M, Benmokhtar S, Talbi M (2013) Removal of a cationic dye from wastewater by adsorption onto natural adsorbents. J Mater Environ Sci 4(1):1-10

Patil MR, Shrivastava VS (2013) Identification of organics by FTIR and GC-MS. Asian J Chem Environ Res 6:22-26

Patil MR, Shrivastava VS (2016) Adsorptive removal of methylene blue from aqueous solution by polyaniline-nickel ferrite nanocomposite: a kinetic approach. Desalination Water Treat 57(13):5879-5887

Periyasamy M, Rajan MR (2009) Physico-chemical characteristics and water quality index of electroplating industry effluent. J Ind Pollut Control 25(1):1-8

Rawway M, Kamel MS, Abdul-Raouf UM (2016) Microbial and physico-chemical assessment of water quality of the River Nile at Assiut Governorate (Upper Egypt). J Ecol Health Environ 14(1):7-14

Saxena U, Saxena S (2015) Correlation study on physico-chemical parameters and quality Assessment of ground water of Bassi Tehsil of district Jaipur, Rajasthan, India, SGVU. Int J Environ Sci Technol 1(1):78-91

Sharma D, Kansal A, Pelletier G (2017) Water quality modeling for urban reach of Yamuna river, India (1999-2009), using QUAL2Kw. Appl Water Sci 7(3):1535-1559

Singariya P, Mourya KK, Gadi BR (2016) Evaluation of Microcidal and Nitrogen assimilatory enzymes activity and identification of $\beta$-sitosterol in C. Environ Impact Biodivers 113-131

Smith CJ, Perfetti TA, Morton MJ, Rodgman A, Garg R, Selassie CD, Hansch C (2002) The relative toxicity of substituted phenols reported in cigarette mainstream smoke. Toxicol Sci 69(1):265-278

Srebrenkoska V, Zezova S, Spasova S, Golomeova S (2014) Methods for waste waters treatment in textile industry. In: International scientific conference "UNITECH 2014", Gabrovo, pp 248-252

Surana AR, Wagh RD (2017) GC-MS profiling and antidepressant-like effect of the extracts of Hamelia patens in animal model. Bangladesh J Pharmacol 12(4):410-416

Vallyathan V, Shi X, Castranova V (1998) Reactive oxygen species: their relation to pneumoconiosis and carcinogenesis. Environ Health Perspect 106(Suppl 5):1151

Wang G, Zhang J, Li X, Bao Z, Liu Y, Liu C, He R, Luo J (2017) Investigating causes of changes in runoff using hydrological simulation approach. Appl Water Sci 7:2245-2253. https://doi.org/10.1007/ s13201-016-0396-1

Publisher's Note Springer Nature remains neutral with regard to jurisdictional claims in published maps and institutional affiliations. 